\title{
The distribution of relative proper motions of wide binaries in GAIA DR2: MOND or multiplicity?
}

\author{
C.J. Clarke ${ }^{1, \star}$ \\ ${ }^{1}$ Institute of Astronomy, Madingley Rd, Cambridge, CBз OHA, UK
}

Submitted:

\begin{abstract}
We examine the distribution of on-sky relative velocities for wide binaries previously assembled from GAIA DR2 data and focus on the origin of the high velocity tail of apparently unbound systems which may be interpreted as evidence for non-Newtonian gravity in the weak field limit. We argue that this tail is instead explicable in terms of a population of hidden triples, i.e. cases where one of the components of the wide binary is itself a close binary unresolved in the GAIA data. In this case the motion of the photocentre of the inner pair relative to its barycentre affects the apparent relative proper motion of the wide pair and can make pairs that are in fact bound appear to be unbound. We show that the general shape of the observed distributions can be reproduced using simple observationally motivated assumptions about the population of hidden triples.
\end{abstract}

Key words: binaries:visual-proper motions- stars:kinematics and dynamics

\section{INTRODUCTION}

It has long been recognised that ultra-wide binaries offer a potential opportunity to scrutinise alternative gravity theories (see e.g. Hernandez et al. (2012), Pittordis \& Sutherland (2018)). This derives from the fact that the gravitational acceleration experienced in solar type binaries with separations of order $10^{4} \mathrm{AU}$ is starting to become comparable to that experienced in the outer reaches of spiral galaxies, where the anomalously large circular velocity provides some of the most convincing evidence for dark matter. Modified gravity theories (MOND, e.g. Milgrom (1983)) instead propose that the law of gravity is modified in the weak field limit. A corollary of this assumption, at least in the case of MOND-like theories that posit a reduced role for the external field effect, is that wide binaries would be subject to a larger gravitational acceleration, resulting in higher orbital speeds, than in the case of purely Newtonian gravity.

Pittordis \& Sutherland (2019) have recently evaluated the evidence for non-Newtonian gravity by examining the kinematics of a large sample of candidate wide binary stars, identified in GAIA DR2 as being stellar pairs with projected separation of $<40000$ A.U., with consistent parallax $<5$ mas (corresponding to an estimated distance of $<200 \mathrm{pc}$ ) and with a relative velocity component in the plane of the sky of $<3 \mathrm{~km} \mathrm{~s}^{-1}$. 'Triples' (i.e. cases where there was evidence for additional resolved companions on a scale $>100$ A.U.) were eliminated from this sample. The normalised velocity

^ E-mail:cclarke@ast.cam.ac.uk (i.e. the on-sky relative velocity normalised to that of a circular binary in the plane of the sky) was calculated for each pair and the distribution of this quantity analysed over four bins of apparent separation in the range $5000-20000$ A.U. In a Newtonian scenario, this distribution should be truncated at a normalised velocity of $\sqrt{2}$, this corresponding to a parabolic orbit in the plane of the sky; pairs with higher normalised velocity would certainly be unbound and would not be expected to be strongly represented in the observed population since the timescale for the change of separation of a parabolic binary at a separation of a few kAU is short, $\sim$ a few $\times 10^{5}$ years. Indeed, although Pittordis \& Sutherland were able to reproduce the distribution of normalised velocities in a Newtonian scenario where hyperbolic fly-bys generated a significant high velocity tail, the required stellar density far exceeded that of the surveyed region.

The key result of the analysis of Pittordis \& Sutherland (2019) is that the distribution of normalised on-sky velocities, while being mainly concentrated at values $<\sqrt{2}$, displayed a prominent 'shelf' (flat high velocity tail) extending up to the cut-off velocity for inclusion in the sample (i.e. typically up to a normalised velocity of $\sim 7$ ). Pittordis \& Sutherland also explored explanations for this high velocity 'shelf' in terms of a variety of alternative gravity theories, finding that while MOND theories without external field effect are incompatible with the data, there is still a potential role for MOND with an external field effect, although the data are not decisive in this regard.

Here we put forward an alternative hypothesis, i.e. that this 'shelf' is a consequence of the higher order multiplic- 
ity of the wide binary sample. Note that the possible influence of hidden multiples has previously been discussed by Hernandez et al. (2012) and also by Pittordis \& Sutherland (2019) although this has not been quantified in detail. In the GAIA data the 'wide' pair is well resolved and, as noted above, systems with evidence of further companions on a scale $>0.5$ mas (typically 100 A.U.) were eliminated from the sample. If the system however contains an unresolved inner binary of non-unit mass ratio, then there is an additional shift of the photocentre of this inner binary with respect to its centre of mass. Consequently this would introduce an error into the derived relative proper of the inner pair and its wide companion. In this paper we generate a synthetic population of wide binaries, including a subset with unresolved components, and evaluate how the shift between the motion of the photocentre and the barycentre of the inner pair can distort the measurements of relative proper motions within wide binaries. Note that in our analysis we only consider inner components with a restricted range of separations: close enough that they are unresolved by GAIA (i.e. $<100$ A.U.) and wide enough that they would not have been eliminated from the sample on the basis of an unacceptable astrometric solution. (Note that inner binaries with separations $<$ a few AU would manifest a variable proper motion over the 22 month baseline of the GAIA DR2 release and would not have been included in the wide sample analysed here).

In Section 2 we present the details of our simulations which use observationally motivated parameters in order to synthesise the effect of higher order multiplicity. Our simulations readily produce a high velocity 'shelf' that is very similar to that observed in the GAIA DR2 data and thus suggest that the observed velocity distribution should not be regarded as prima facie evidence for non- Newtonian gravity. We point out that with a more exact definition of the separation limits for the inner binaries that are hidden in the DR2 sample, the observed distribution of normalised velocities instead provides a tool for characterising higher order multiplicity within wide solar type binaries.

\section{THE EFFECT OF HIGHER ORDER MULTIPLICITY ON THE NORMALISED VELOCITY DISTRIBUTION}

\subsection{Simulation method}

In order to assess the effect of concealed inner binaries on wide binary kinematics, we construct a fiducial model, focusing on wide binaries with a solar type primary and separations on the sky in the four logarithmically spaced bins considered by Pittordis \& Sutherland (2019) in the range $5000-20000$ A.U., using the inter-bin variation in the numbers of binaries to assign the probability distribution within each bin. The components of the wide binaries are selected at random from a distribution with mass ratio, $q$, where the fraction of systems per unit interval of mass ratio scales as $q^{-1.1}$ (Moe \& Di Stefano 2017); given the magnitude limits imposed by Pittordis \& Sutherland we only consider outer pairs with a mass ratio in the range $0.5-1$. Such binaries are uniformly distributed in $\cos i$ (where $i$ is the angle between the line of sight and the normal to the binary orbit). The binary eccentricities are selected from a thermal distribution (fraction per unit interval of eccentricity, $e$, scaling as $e$ (Heggie 1975)) and the relative velocity between the components of the wide pair in the plane of the sky is calculated. As expected, this gives rise to a distribution that truncates at normalised velocity ratio of $\sqrt{2}$ and matches the distributions in the Newtonian case shown in Pittordis \& Sutherland (2019).

We then furthermore assume that a fraction $f_{\text {triple }}$ of these wide pairs have a primary or secondary component that is itself a binary with separation $a$, that is uniformly distributed in log separation over the range $a_{\min }$ to $a_{\max }$. As noted above, $a_{\min }$ has to be large enough that the astrometric solution of the component was not flagged as exhibiting variability over the duration of the GAIA DR2 experiment. $a_{\max }$ has to be small enough that the inner pair was not resolved by GAIA and rejected as a 'triple' from the sample. We here adopt $a_{\min }=3$ A.U. and $a_{\max }=100$ A.U. based on the 22 month duration of the GAIA DR2 dataset and the resolution limits for the sample quoted by Pittordis \& Sutherland (2019). We assume that the orbital plane of each inner binary is randomly inclined with respect to the plane of the outer binary and that the mass ratio and eccentricity distribution are randomly selected from the same distributions given above for generating the outer pairs, with the mass ratio distribution extending down to $q=0.1 .{ }^{1}$ The components of the inner binary are then assigned $\mathrm{G}$ band magnitudes using the mass luminosity relationship given by equation (3) of Pittordis \& Sutherland (2019), and from this the on sky motion of the inner binary's photocentre relative to its barycentre is calculated. For such hidden triples, the relative proper motion of the two wide components is evaluated as the velocity in the sky plane of the inner binary's photocentre with respect to its distant companion. Binaries and hidden triples are then treated identically ${ }^{2}$ in that the normalised velocity (i.e. the ratio of the on-sky relative motion to that of a circular binary in the plane of the sky) is calculated for each wide system, thus generating a distribution over the synthesied population. We adopt $f_{\text {triple }}=0.5$, motivated by the results of Riddle et al. (2015) who found that in a sample of 212 wide binaries, 100 had additional inner components with separa-

1 There is some evidence that the mass ratio distribution becomes more weighted towards higher $q$ for small separations, although the errorbars on mass ratio distribution slopes are large and in any case are not evaluated specifically for the inner pairs of multiples. In order to investigate the sensitivity of the resulting velocity distributions on the assumed mass ratio distribution we have also considered, for inner binaries, the mass ratio distribution quoted by Moe \& di Stefano for binaries with separations around 1 AU: these are parameterised with power law slopes of -0.1 and -0.5 in the domains respectively below and above $q=0.3$. This change slightly boosts the perturbations associated with hidden triple components as it gives more prominence to pairs of intermediate $(q \sim 0.6 \pm 0.3)$ for which the photocentrebarycentre motion is maximal; the effect is however small $(<10 \%$ in average normalised photocentre shift). We thus conclude that the results are robust against plausible variations in the assumed mass ratio distribution.

2 We have also considered the small modification associated with the fact that the observer who cannot resolve the inner pair will under-estimate its mass from the mass-luminosity relation: this however only changes the circular velocity assigned by of order $1 \%$. 
tions in the range of a few to a few hundred A.U. (see also Tokovinin (2014) and Halbwachs et al. (2017) for evidence of a high inner multiple fraction within wide pairs).

\subsection{Simulated results}

Figure 1 presents the results of the above described fiducial model in each of the separation bins considered by Pittordis $\&$ Sutherland. We compare the predictions of this model for the distribution of normalised velocities (dashed) to the corresponding distributions observed in each bin (solid), using the sub-samples of the GAIA DR2 data for which the relative velocity error is less than 0.25 times the circular velocity. The number of synthesised binaries matches the sample number in each separation bin (respectively 629, 428, 270 and 134). It can be seen that the simulated populations recover the main features of the observed distribution in all separation bins without any fine tuning of the models, a point that is emphasised by the excellent agreement between the corresponding cumulative distributions shown in Figure 2 (we have checked that the small deviations between the synthesised and observed cumulative distributions seen in Figure 2 are not statistically significant given the sample sizes involved).

While the majority of the population has normalised velocity $<\sqrt{2}$ there is a significant tail to higher velocities which is entirely composed of hidden triple systems where the relative motion of the photocentre and barycentre of the unresolved inner pair is sufficient to scatter bound systems so that they appear to be unbound in this plot. It can readily be seen how the magnitude of relative motion between the barycentre and photocentre of an unresolved pair can affect the apparent kinematics of wide binaries. For example, the relative velocity of a solar mass circular binary of separation 10 A.U. is around $10 \mathrm{~km} \mathrm{~s}^{-1}$; if the internal mass ratio of this binary were 0.25 , for example, the relative velocity between the barycentre and photocentre would be $\sim 2 \mathrm{~km} \mathrm{~s}^{-1}$ which is a significant value compared with the circular velocity of the wide $(\sim 5000$ A.U. $)$ binary $\left(\sim 0.5 \mathrm{~km} \mathrm{~s}^{-1}\right)$. The featureless nature of this excess at high normalised velocities stems from the combined effects of a broad distribution of mutual inclinations, ecentricities and mass ratios of the inner pair.

We also note the consistency between the model predictions and observations concerning trends with increasing binary separation. In the low error sub-samples analysed in Pittordis \& Sutherland, the number of systems with normalised velocity ratio $>\sqrt{2}$ is 159 in the separation range 5000-7000 A.U. and 53 in the separation range 14000-20000 A.U.. As the authors note, an explanation in terms of flybys would instead imply an increase in the absolute number of such objects at large separation. In the framework of hidden triples, however, the velocity component associated with a given population of close pairs would represent an increasing fraction of the circular velocity of the distant pair at large separations and so would imply that the fraction of wide pairs with high normalised velocity ratios should increase at large separations. Observationally this is indeed the case (e.g. $159 / 629 \sim 0.25$ pairs in the $5000-7000$ A.U. range have normalised velocities $>\sqrt{2}$ compared with the corresponding fraction 53/134 0.4 in the $5000-7000$ A.U. range.)
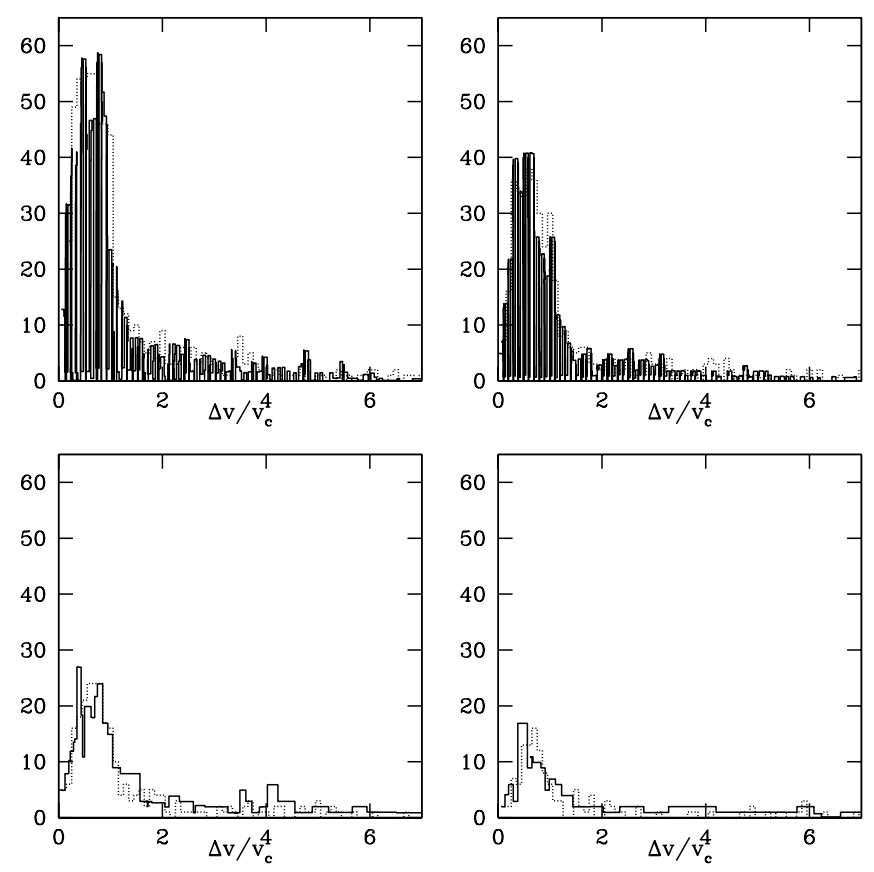

Figure 1. Normalised velocity distribution for the fiducial model (dashed: see Section 2.1) compared with data (solid) in the separation ranges: 5000- 7000 A.U. (upper left), 7000-10000 A.U. ( upper right), 10000-14000 A.U. (lower left) and 14000-20000 A.U. (lower right) from Pittordis \& Sutherland (2019).
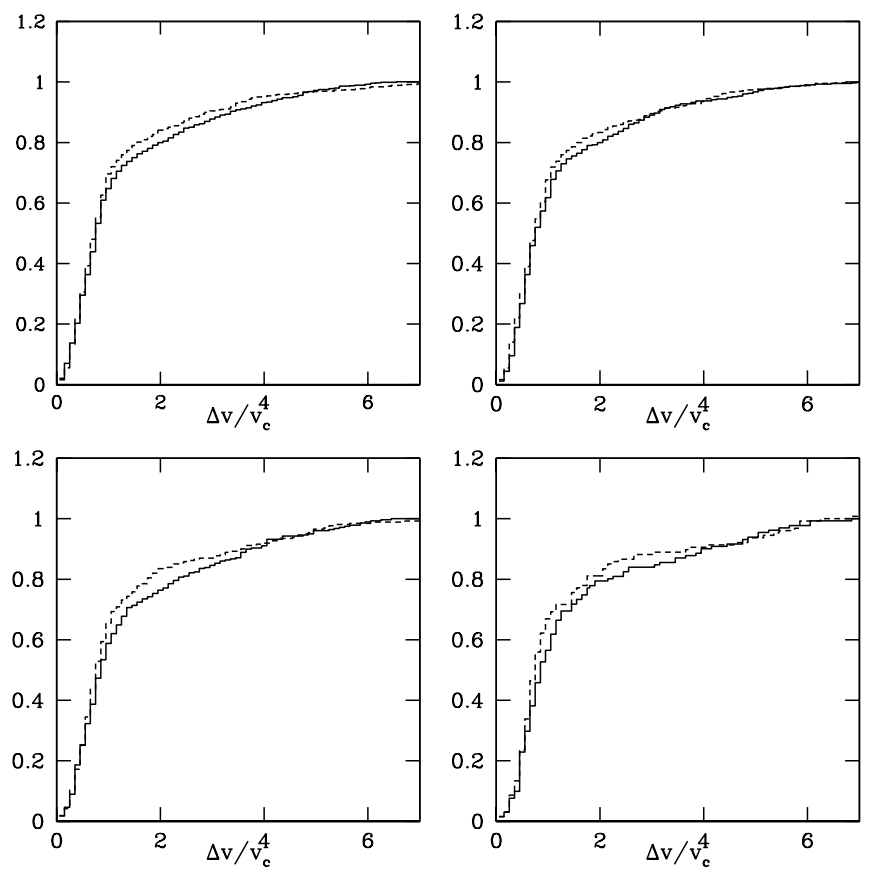

Figure 2. Cumulative distributions corresponding to histograms shown in Figure 1. 


\section{$4 \quad$ Clarke}

\section{CONCLUSIONS}

We have shown that the observed population of wide binaries with normalised velocities in excess of $\sqrt{2}$ as derived from GAIA DR2 data is readily explicable in terms of contamination of the sample by unresolved close ( a few to $\sim 100$ A.U.) inner multiple components. Such 'hidden triples' introduce an error in the kinematics of the wide pair which stems from the relative motion of the barycentre and photocentre of the inner pair. In consequence some wide systems can appear to be unbound when in fact they are bound hierarchical multiple systems governed by Newtonian dynamics.

We find that the 'shelf' of high velocity ratios can be entirely attributable to hidden triples if the triple fraction is as high $(\sim 50 \%)$ as suggested by local surveys (Riddle et al. 2015). This is clearly a hypothesis that can be tested by targeted investigations (e.g. high contrast adaptive optics imaging) of objects with anomalously high velocity ratios. Likewise the hidden triple hypothesis would imply possible anomalies in the locations of stars in the colour magnitude diagram (e.g. Widmark et al. (2018))Hidden triples can also generate discrepant proper motions over longer time baselines. We note that our fiducial model predicts that, when comparing proper motions of wide binaries obtained from the ten year Hipparcos survey and the 22 month DR2 survey, $<10 \%$ of the sample would be expected to show discordant proper motions at a level comparable with typical Hipparcos errors. Such a figure is compatible with the comparison presented in Hernandez et al. (2019).

The important contribution from hidden triples raises a caution against interpreting this population of apparently unbound pairs as evidence for a modification of the gravitational acceleration in the weak gravity regime (i.e. as evidence for MOND or a variant theory). At the very least, further examination of this subject will need to examine a subset of proper motion data where there are independent limits on the existence of inner binary components. Conversely, if retaining the assumption of Newtonian gravity, GAIA data has the possibility to provide statistical constraints on higher order multiplicity on samples that are far larger than those assessed in conventional multiplicity surveys.

\section{ACKNOWLEDGMENTS}

I am grateful to useful input from Will Sutherland and to the referee for comments which have improved the paper.

\section{REFERENCES}

Halbwachs J. L., Mayor M., Udry S., 2017, MNRAS, 464, 4966 Heggie D. C., 1975, MNRAS, 173, 729

Hernandez X., Jiménez M. A., Allen C., 2012, in Journal of Physics Conference Series. p. 012018 (arXiv:1205.5767), doi:10.1088/1742-6596/405/1/012018

Hernandez X., Cortés R. A. M., Allen C., Scarpa R., 2019, International Journal of Modern Physics D, 28, 1950101

Milgrom M., 1983, ApJ, 270, 365

Moe M., Di Stefano R., 2017, ApJS, 230, 15

Pittordis C., Sutherland W., 2018, MNRAS, 480, 1778

Pittordis C., Sutherland W., 2019, MNRAS, 488, 4740

Riddle R. L., et al., 2015, ApJ, 799, 4
Tokovinin A., 2014, AJ, 147, 87

Widmark A., Leistedt B., Hogg D. W., 2018, ApJ, 857, 114 